International Journal of Modern Physics A,

(C) World Scientific Publishing Company

\title{
UPPER BOUND ON THE SCALE OF MAJORANA-NEUTRINO MASS GENERATION*
}

\author{
J. M. NICZYPORUK \\ Department of Physics, University of Illinois at Urbana-Champaign \\ 1110 West Green Street, Urbana, Illinois 61801-3080, USA
}

\begin{abstract}
We derive a model-independent upper bound on the scale of Majorana-neutrino mass generation. The upper bound is $4 \pi v^{2} / \sqrt{3} m_{\nu}$, where $v \simeq 246 \mathrm{GeV}$ is the weak scale and $m_{\nu}$ is the Majorana neutrino mass. For neutrino masses implied by neutrino oscillation experiments, all but one of these bounds are less than the Planck scale, and they are all within a few orders of magnitude of the grand-unification scale.
\end{abstract}

Considerable evidence has mounted that one or more of the three neutrino species has a nonzero mass, based on the observation of neutrino oscillations. 1 Since neutrino masses are necessarily associated with physics beyond the Standard Model, one would like to know the energy scale at which this new physics resides. In this talk (see Ref. 2 for further details), we present a model-independent upper bound on the scale of Majorana-neutrino mass generation.

We assume that the neutrino masses are Majorana, unlike the other known fermions, which carry electric charge and are therefore forbidden to have Majorana masses. If there is no $\mathrm{SU}(2)_{L} \times \mathrm{U}(1)_{Y}$-singlet fermion field in nature, then neutrino masses are necessarily Majorana. However even if such a field exists, its mass is naturally much greater than the weak scale 3 in which case light neutrinos are Majorana fermions. In the Standard Model, Majorana neutrino masses are forbidden by an "accidental" global $B-L$ symmetry (baryon number minus lepton number), but there is no reason to expect that this symmetry is fundamental.

We begin our analysis with the Standard Model, but with a Majorana neutrino mass of unspecified origin. Since the neutrino mass is put in artificially, this is only an effective field theory, valid up to some energy scale at which it is subsumed by a deeper theory, which we regard as the scale of Majorana-neutrino mass generation. A simple way to derive an upper bound on the scale at which the effective theory breaks down is to impose unitarity on the tree-level $2 \rightarrow 2$ scattering amplitudes that grow with energy. 1 In the high-energy limit, $s \gg M_{W}^{2}, M_{Z}^{2}, m_{\nu}^{2}, m_{\ell}^{2}$, the relevant zeroth-partial-wave amplitudes (see Fig. 1) are given by

$$
\begin{aligned}
a_{0}\left(\frac{1}{\sqrt{2}} \nu_{i \pm} \nu_{j \pm} \rightarrow W_{L}^{+} W_{L}^{-}\right) & \sim \mp \frac{m_{\nu_{i}} \sqrt{s}}{8 \pi \sqrt{2} v^{2}} \delta_{i j} \\
a_{0}\left(\frac{1}{\sqrt{2}} \nu_{i \pm} \nu_{j \pm} \rightarrow \frac{1}{\sqrt{2}} Z_{L}^{0} Z_{L}^{0}\right) & \sim \mp \frac{m_{\nu_{i}} \sqrt{s}}{8 \pi v^{2}} \delta_{i j}
\end{aligned}
$$

*Talk presented at DPF 2000, Ohio State Univ., Columbus, OH, Aug. 9-12, 2000. 

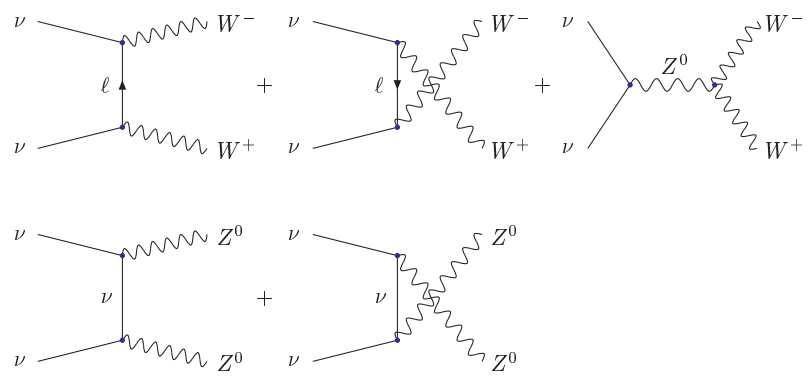

Figure 1: Feynman diagrams that contribute to the amplitudes in Eqs. (1)-(2). The source of the Majorana neutrino mass is unspecified, so there are no diagrams involving the coupling of the Majorana neutrino to the Higgs boson. Unitary gauge is used throughout.

where $v=\left(\sqrt{2} G_{F}\right)^{-1 / 2} \simeq 246 \mathrm{GeV}$ is the weak scale, the indices $i, j$ denote the three neutrino mass eigenstates, the subscripts on the neutrinos and charged leptons indicate helicity $\pm 1 / 2$, and the subscript on the partial-wave amplitude indicates $J=0$.

The strongest bound on the scale of Majorana-neutrino mass generation is obtained by considering a scattering process which is a linear combination of the above amplitudes:

$$
a_{0}\left(\frac{1}{2}\left(\nu_{i+} \nu_{i+}-\nu_{i-} \nu_{i-}\right) \rightarrow \frac{1}{\sqrt{3}}\left(W_{L}^{+} W_{L}^{-}+Z_{L}^{0} Z_{L}^{0}\right)\right) \sim-\frac{\sqrt{3} m_{\nu_{i}} \sqrt{s}}{8 \pi v^{2}} .
$$

The unitarity condition on inelastic $2 \rightarrow 2$ scattering amplitudes, $\left|a_{J}\right| \leq 1 / 2$, 目 implies that the scale of Majorana-neutrino mass generation is less than the scale

$$
\Lambda_{M a j} \equiv \frac{4 \pi v^{2}}{\sqrt{3} m_{\nu}}
$$

which is inversely proportional to the neutrino mass. This is our principal result.

We have analyzed 2 two explicit models of light Majorana-neutrino masses that exemplify this bound: one with a heavy right-handed neutrino $\nu_{R}$ (see-saw mechanism), and one with a heavy $\mathrm{SU}(2)_{L}$-triplet Higgs field. Unitarity is restored by the heavy Majorana neutrino $N \approx \nu_{R}$ in the see-saw model and by the heavy Higgs scalars in the triplet Higgs model, whose masses respect the bound in Eq. (仹). In both cases, the bound is saturated when dimensionless parameters in these models (Yukawa couplings and/or mass ratios) attain their maximum values. Indeed, these models can naturally saturate our bound, Eq. (4), precisely because they generate a Majorana-neutrino mass term of dimension five in the low-energy theory. In general, saturating unitarity-based bounds is nontrivial; for example, there is no known model that saturates the upper bound on the scale of Dirac-fermion mass generation 4 . 
Table 1: Neutrino mass-squared differences from a variety of neutrino oscillation experiments, and their interpretations. The last column gives the upper bound on the scale of Majorana-neutrino mass generation, Eq. (11), for each interpretation. Table adapted from Ref. 1.

\begin{tabular}{lllc}
\hline \hline Experiment & Favored Channel & $\Delta m^{2}\left(\mathrm{eV}^{2}\right)$ & $\Lambda_{M a j}(\mathrm{GeV})<$ \\
\hline LSND & $\bar{\nu}_{\mu} \rightarrow \bar{\nu}_{e}$ & $0.2-2.0$ & $9.8 \times 10^{14}$ \\
Atmospheric & $\nu_{\mu} \rightarrow \nu_{\tau}$ & $3.5 \times 10^{-3}$ & $7.4 \times 10^{15}$ \\
Solar & & & \\
MSW (large angle) & $\nu_{e} \rightarrow \nu_{\mu}$ or $\nu_{\tau}$ & $(1.3-18) \times 10^{-5}$ & $1.2 \times 10^{17}$ \\
MSW (small angle) & $\nu_{e} \rightarrow$ anything & $(0.4-1) \times 10^{-5}$ & $2.2 \times 10^{17}$ \\
Vacuum & $\nu_{e} \rightarrow \nu_{\mu}$ or $\nu_{\tau}$ & $(0.05-5) \times 10^{-10}$ & $2.0 \times 10^{20}$ \\
\hline \hline
\end{tabular}

Neutrino oscillation experiments do not measure the neutrino mass, but rather the absolute value of the mass-squared difference of two species of neutrinos, $\Delta m^{2}$. This implies a lower bound of $m_{\nu} \geq \sqrt{\Delta m^{2}}$ on the mass of one of the two participating neutrino species. Using Eq. (4), one finds the upper bounds on the scale $\Lambda_{M a j}$ given in Table 1 for a variety of neutrino oscillation experiments. These upper bounds are all within a few orders of magnitude of the Planck scale, $G_{N}^{-1 / 2} \simeq$ $1.2 \times 10^{19} \mathrm{GeV}$, which is the scale before which quantum gravity must become relevant. However, only the vacuum-oscillation interpretation of the solar neutrino deficit yields a scale that could be as large as the Planck scale. In all other cases, we find that the physics of Majorana-neutrino mass generation must be below the Planck scale. Thus, if these neutrino masses arise from quantum gravity, then the scale of quantum gravity must be somewhat less than the Planck scale.

The upper bounds on $\Lambda_{M a j}$ are also within a few orders of magnitude of the grand-unification scale, $\mathcal{O}\left(10^{16}\right) \mathrm{GeV}$. The LSND and atmospheric neutrino experiments yield an upper bound on $\Lambda_{M a j}$ slightly below the grand-unification scale, but the scale of Majorana-neutrino mass generation could be less than the unification scale in a grand-unified model. For example, in a grand-unified model that makes use of the see-saw mechanism, the mass of the heavy Majorana neutrino $N$ could be equal to a small Yukawa coupling times the vacuum-expectation value of the Higgs field that breaks the grand-unified group.

\section{References}

1. R. G. Robertson, hep-ex/0001034 in Proc. of the 19th Intl. Symp. on Lepton and Photon Interactions at High Energies (Lepton-Photon 99), Stanford, CA, 1999.

2. F. Maltoni, J. M. Niczyporuk, and S. Willenbrock, hep-ph/0006358.

3. H. Georgi, Nucl. Phys. B156, 126 (1979).

4. T. Appelquist and M. Chanowitz, Phys. Rev. Lett. 59, 2405 (1987).

5. W. Marciano, G. Valencia, and S. Willenbrock, Phys. Rev. D 40, 1725 (1989).

6. M. Golden, Phys. Lett. B 338, 295 (1994); S. Jäger and S. Willenbrock, Phys. Lett. B 435, 139 (1998); R. S. Chivukula, Phys. Lett. B 439, 389 (1998). 